\title{
Salt-induced protein resistance of polyelectrolyte brushes studied using fluorescence correlation spectroscopy and neutron reflectometry $\dagger$
}

\author{
C. Czeslik, ${ }^{{ }^{a}}$ G. Jackler, ${ }^{a}$ T. Hazlett, ${ }^{b}$ E. Gratton, ${ }^{b}$ R. Steitz, ${ }^{c}$ A. Wittemann ${ }^{d}$ and \\ M. Ballauff ${ }^{d}$ \\ a Universität Dortmund, Physikalische Chemie I, D-44221 Dortmund, Germany. \\ E-mail: claus.czeslik@uni-dortmund.de; Fax: +49 231755 3901; Tel: +49 2317553903 \\ ${ }^{b}$ University of Illinois, Laboratory for Fluorescence Dynamics, Urbana, Illinois 61801, USA \\ ${ }^{c}$ Hahn-Meitner-Institut Berlin, D-14109 Berlin, Germany \\ ${ }^{d}$ Universität Bayreuth, Physikalische Chemie I, D-95440 Bayreuth, Germany
}

\begin{abstract}
We used two-photon excitation fluorescence correlation spectroscopy (FCS) and neutron reflectometry to study in situ the effect of salt concentration on the degree of protein binding to polyelectrolyte brushes. The binding of bovine serum albumin (BSA) to poly(acrylic acid) (PAA) brushes was characterized at neutral pH values where both the protein and the brushes carry a negative charge. Spherical PAA brush particles were used in the FCS experiments, whereas a planar PAA brush served as protein substrate in the neutron reflectometry experiments. It has been found that BSA binds strongly to both the spherical and the planar PAA brushes under electrostatic repulsion at low ionic strength. The BSA volume fraction profile, as determined from the neutron reflectivities, indicates a deep penetration of the BSA molecules into the PAA brush. However, the analysis of the FCS data reveals that the protein affinity of the spherical PAA brush particles decreases drastically when increasing the concentration of sodium chloride to a few $100 \mathrm{mM}$. This observation is in line with the measured neutron reflectivities of the planar PAA brush. The reflectivity curve obtained in the absence of protein is virtually overlapping with that measured when the PAA brush is in contact with a BSA solution but containing $500 \mathrm{mM}$ sodium chloride which suggests protein resistance of the planar PAA brush at this elevated salt concentration. The results of this study provide evidence for a new kind of protein-resistant interfaces. Whereas protein binding to the PAA brush is likely to be dominated by the release of counterions, this driving force vanishes as the ionic strength of the solution is raised and protein molecules are repelled from the interface by steric interactions. In a general view, the "switching" of the protein affinity of a PAA brush by varying the ionic strength of the protein solution over a relatively small range may appear to be useful for biotechnological applications.
\end{abstract}

\section{Introduction}

The adsorption of protein molecules at interfaces is one of the most interesting subjects in biophysical research, as it is the basis for a broad range of processes occurring in nature and biotechnology. ${ }^{4}$ Examples are the activity of enzymes at biological membranes, the stabilization of food emulsions, or the use of solid-phase immunoassays in medical diagnostics. As a rule that has emerged from numerous studies on protein adsorption carried out so far, one may generalize that all proteins adsorb at all interfaces. This rule originates largely in the heterogeneity of the protein surface and the conformational flexibility of the protein structure. As a result, protein molecules can interact with interfaces via direct ionic, van der Waals, and hydrophobic forces which are often enhanced by interface-induced conformational changes of the protein molecules. ${ }^{59}$ However, there is no rule without exceptions. The search for and the understanding of protein-resistant interfaces has also attracted much research interest, since protein adsorption may have unfavourable consequences, such as biofilm formation on used contact lenses, biofouling of medical implants, or the attachment of mussels at ships. 3,10

† Presented at the annual meeting of the Deutsche Bunsen-Gesellschaft für Physikalische Chemie, Dresden, Germany, May 20-22, 2004.
The most prominent example of a protein-resistant surface coating is a brush of poly(ethylene oxide) (PEO) where one end of the PEO chains is chemically bound to the solid substrate. ${ }^{11}$ 15 The protein resistance of such brushes may be explained by steric repulsions. When a protein molecule approaches a brush of hydrophilic long flexible polymer chains, the brush will be compressed leading to an unfavourable reduced conformational entropy of the polymer chains. Protein resistance is also observed for surfaces covered by dextran ${ }^{16,17}$ and for a series of self-assembled monolayers carrying different chemical groups. ${ }^{18,19}$ It is interesting to note that PEO chains having only a few monomers are also effective in rejecting protein molecules. ${ }^{18} 20$ It is argued that the strong interaction of the sorbent surface with water is causing protein resistance, because it prevents direct contact between the surface and the protein. $18,21,22$

In a recent study, the binding of bovine serum albumin (BSA) to spherical polyelectrolyte brush (SPB) particles was characterized. ${ }^{23}$ The particles consist of a solid core of poly(styrene) (PS) onto which long linear poly(acrylic acid) (PAA) chains are grafted. ${ }^{24,25}$ The adsorbed amount of protein was measured ex situ, i.e., in the absence of non-adsorbed protein. Weakly and non-adsorbed BSA was removed from the BSA/SPB suspension by ultrafiltration, and the protein concentration of the filtrate was determined using UV spectroscopy. It has been found that the SPB particles bind huge 
amounts of BSA under electrostatic repulsion at low ionic strength. ${ }^{23}$ Moreover, BSA that is irreversibly adsorbed at low ionic strength can be desorbed from the SPB particles by rinsing with a $500 \mathrm{mM}$ sodium chloride solution. This observation is rather unexpected, since the elevated ionic strength is shielding the direct electrostatic repulsion between BSA and the SPB particles. Thus, a PAA brush provides a unique substrate for protein molecules showing a strongly reduced protein affinity when the salt concentration of the protein solution is raised to a few $100 \mathrm{mM}$. It is interesting to note that SPB particles provide a mild environment for protein molecules, since the conformation and the enzymatic activity of immobilized proteins have been found to be largely preserved. ${ }^{26-28}$

In view of the highly interesting properties of the spherical PAA brush particles in terms of protein adsorption, the main aim of this study was to characterize and compare the degree of BSA binding to spherical and planar PAA brushes in situ. Here we present for the first time a quantitative analysis of the saltinduced protein resistance of PAA brushes by using fluorescence correlation spectroscopy (FCS) and neutron relflectometry. FCS was applied to BSA/SPB suspensions as a function of ionic strength. In contrast to an earlier study where fluorescence fluctuation data have been evaluated by calculating photon counting histograms, ${ }^{29}$ autocorrelation functions as derived from fluorescence fluctuation data are used in this study to quantify the effect of salt on the degree of BSA binding to SPB particles. The autocorrelation function of a multi-component system can be decomposed, if the components have markedly different diffusion constants. ${ }^{30}$ For the system under study, two components are relevant, the free non-adsorbed protein molecules with a large diffusion constant in the presence of the slowly diffusing SPB particles with adsorbed protein molecules. As has been shown by using neutron reflectometry, BSA binds to a planar PAA brush at low ionic strength only. ${ }^{31}$ Here we give an extended analysis of these experiments and illustrate for the first time the effect of the ionic strength on the volume fraction profile of BSA across a planar PAA brush. Neutron reflectometry is the most powerful tool to determine the structure of a planar solid/liquid interface that is interacting with protein molecules, since neutrons can penetrate condensed matter over macroscopic distances, contrast variation is easily achieved by $\mathrm{D}_{2} \mathrm{O} / \mathrm{H}_{2} \mathrm{O}$ exchange, and the interfacial structure can be obtained with $\AA$ resolution. ${ }^{32}$ BSA was chosen as model protein, since it is characterized by a relatively low isoelectric point of about 5 and thus carries a net negative charge at neutral $\mathrm{pH}$-values. ${ }^{33}$ As a consequence, direct ionic interactions between the protein molecules and a PAA brush may be ruled out as the main driving force for BSA adsorption at a PAA brush. As will be shown in this study, BSA binds strongly to and penetrates deeply into a PAA brush at low ionic strength, whereas the adsorption of BSA is suppressed efficiently in the presence of a few $100 \mathrm{mM}$ sodium chloride. Since this saltinduced protein resistance of a PAA brush cannot be related to a simple electrostatic screening, a PAA brush may be regarded as a new type of protein sorbent surface.

\section{Experimental}

\section{Sample preparation}

BSA was purchased from Sigma (catalog number A-6003). It was analysed by gel electrophoresis and was found to be essentially pure. For the FCS experiments, BSA was labelled with Texas Red dye (Molecular Probes) by adding the dye to BSA dissolved in carbonate buffer $(100 \mathrm{mM}, \mathrm{pH}=8.4)$. The dye was covalently bound to BSA as an amine-reactive succinimidyl ester. After about $60 \mathrm{~min}$, unbound dye molecules were removed from the BSA solution using a Sephadex G-25 column which was rinsed with a morpholinoethanesulfonic acid (MES) buffer (10 mM, pH =6.1). From UV spectroscopy of the purified stock solution, a degree of labelling of $91 \%$ and a BSA concentration of $1.1 \mu \mathrm{M}$ were determined. The degree of labelling was calculated as the molar ratio of dye to protein. The spherical PAA brush particles that serve as substrate for BSA in the FCS experiments have a PS core and a PAA brush shell. They were prepared by photoemulsion polymerisation as described before. ${ }^{34}$ The radius of the core is $51 \mathrm{~nm}$, the contour length of the PAA chains is $80 \mathrm{~nm}$ (weight average) and the grafting density is $0.13 \mathrm{~nm}^{-2} \cdot{ }^{23}$ SPB stock solutions of $1 \mu \mathrm{g}$ $\mu \mathrm{L}^{-1}$ in MES buffer were prepared. Then, appropriate amounts of the BSA and the SPB stock solutions were added to a MES buffer solution containing different concentrations of sodium chloride. After intensive mixing, the samples were equilibrated for at least $1 \mathrm{~h}$ before measurement.

For the neutron reflectometry experiments, a $10 \mathrm{mM}$ MES buffer solution was prepared in $\mathrm{D}_{2} \mathrm{O}$ and adjusted to $\mathrm{pD}=6.9$. Using this buffer solution, a BSA stock solution of $1 \mathrm{mg} \mathrm{ml}^{-1}$ was made. Final sample solutions were prepared by mixing 0.8 $\mathrm{ml}$ BSA stock solution with $15.2 \mathrm{ml} \mathrm{D}_{2} \mathrm{O}$ buffer solution. For contrast variation, $0.8 \mathrm{ml}$ BSA stock solution, $11.2 \mathrm{ml} \mathrm{D}_{2} \mathrm{O}$ buffer solution and $4 \mathrm{ml} \mathrm{H}_{2} \mathrm{O}$ buffer solution were mixed instead. The $3: 1 \mathrm{D}_{2} \mathrm{O} / \mathrm{H}_{2} \mathrm{O}$ solvent will be denoted below as HDO. The BSA concentration was always $0.05 \mathrm{mg} \mathrm{ml}^{-1}$. The Si wafers that served as solid support for planar PAA brushes were purchased from Siliciumbearbeitung Andrea Holm (Tann, Germany). They have a size of $8 \times 5 \times 1.5 \mathrm{~cm}$ with the two large sides being polished. The wafers were cleaned by heating in a $1: 1: 5$ mixture of $\mathrm{H}_{2} \mathrm{O}_{2}(30 \%), \mathrm{NH}_{3}(30 \%)$ and $\mathrm{H}_{2} \mathrm{O}$ followed by intensive rinsing with pure water (modified RCA cleaning procedure). ${ }^{35}$ The preparation of a planar PAA brush that was used in the neutron reflectometry experiments was performed according to the literature with slight modifications. ${ }^{36}$ A thin film of perdeuterated PS (d-PS) from Polymer Standards Service (Mainz, Germany) was deposited on one of the large sides $(8 \times 5 \mathrm{~cm})$ of a clean Si wafer by spin coating at $4000 \mathrm{rpm}$ using a $5.5 \mathrm{mg} \mathrm{ml}^{-1}$ polymer solution in toluene (spin coater KW-4A from Chemat Technology). A film thickness of about $170 \AA$ is obtained in this way which was measured by neutron reflectometry as described below (Fig. 1). $5 \mathrm{mg}$ of the diblock copolymer PS-PAA from Polymer Source (Dorval, Canada) were dissolved in $3 \mathrm{ml} \mathrm{1,4-dioxane} \mathrm{at} 60{ }^{\circ} \mathrm{C}$. The copolymer consisted of 34 styrene and 160 acrylic acid monomers. After addition of $2 \mathrm{ml}$ toluene, the PS-PAA solution was spread on a water surface in a Langmuir trough to generate a PS-PAA monolayer with a packing density of 0.1 chains per $\mathrm{nm}^{2}$. The water contained $1 \mathrm{mM} \mathrm{NaCl}$ and was adjusted to $\mathrm{pH}=4.7$ with concentrated $\mathrm{HCl}$ solution to lower the charge of the copolymer and to stabilize the copolymer monolayer on the water surface. Applying the Langmuir-Schaefer

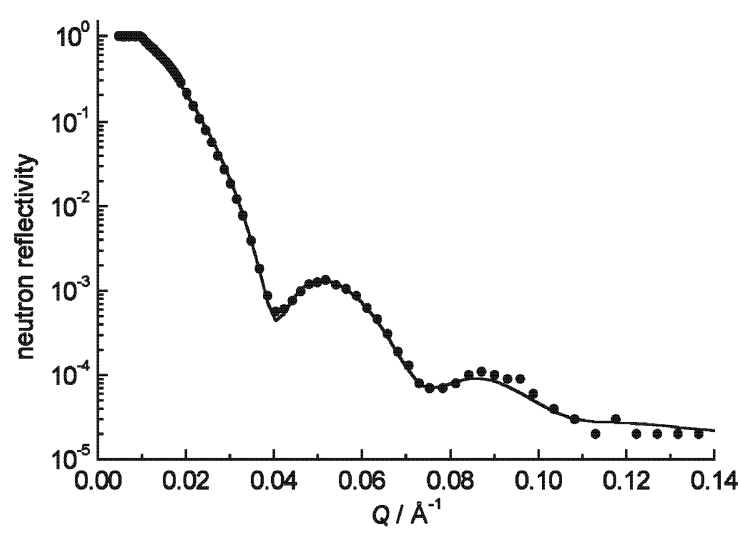

Fig. 1 Neutron reflectivity curve of an air/d-PS/Si interface. The symbols represent the experimental data, the solid line represents a fit based on a one-layer model for the interfacial structure. The data shown are consistent with a layer thickness of $173 \AA$ for the d-PS film. 
technique, ${ }^{37}$ a d-PS coated Si wafer was dipped into the water to transfer PS-PAA molecules to the $\mathrm{Si}$ wafer. From the changes in surface pressure, a transfer ratio of $1.5 \pm 0.3$ was calculated for three different wafers corresponding to a grafting density $0.15 \mathrm{~nm}^{-2}$. Finally, the wafers were heated at 110 ${ }^{\circ} \mathrm{C}$ for 10 min which leads to a diffusion of the PS chains of the PS-PAA molecules into the d-PS films thereby creating stable and irreversibly fixed PAA brushes. ${ }^{36}$

\section{Methods}

The two-photon excitation FCS experiments were carried out at the Laboratory for Fluorescence Dynamics (LFD) at the University of Illinois at Urbana-Champaign (Urbana, IL, USA). A Zeiss Axiovert 135 TV inverted microscope with a Zeiss F Fluar $40 \times / 1.30$ oil objective was used. The excitation light source was a Coherent Mira 900 mode-locked $\mathrm{Ti}$ : sapphire laser which was pumped by a Coherent Verdi cw laser $(532 \mathrm{~nm}, 5 \mathrm{~W})$. The excitation light had a wavelength of $780 \mathrm{~nm}$, a pulse frequency of $80 \mathrm{MHz}$ and a pulse width of about 150 fs at the sample. An avalanche photodiode (EG\&G, model SPCM-AQR-15) was used as the fluorescence detector. The output of the photodiode was directly read into a homebuilt (LFD) computer acquisition card and stored in memory. The photon counts were sampled at $20 \mathrm{kHz}$. Data were processed and analysed with SimFCS and Globals Unlimited ${ }^{\mathrm{TM}}$ software packages developed at the LFD. A three dimensional Gaussian point spread function was assumed which was calibrated using an $11 \mathrm{nM}$ fluorescein solution at $\mathrm{pH}>9$ (the diffusion constant is $300 \mu \mathrm{m}^{2} \mathrm{~s}^{-1}$ ). All FCS experiments were performed three or four times.

The neutron reflectometry measurements were performed at the Hahn-Meitner-Institut in Berlin (Germany) using the V6 instrument. ${ }^{38}$ The neutron wavelength selected by a graphite monochromator was $4.66 \AA$. Higher-order wavelengths were suppressed by a Be filter cooled with liquid nitrogen. The sample cell consisted of the $\mathrm{Si}$ wafer modified with a PAA brush and a Teflon trough, which was fixed to the Si wafer and was filled with the sample solution. The neutrons entered the Si wafer through one of the small sides, were reflected in part at the Si/PAA brush/solution interface, and left the $\mathrm{Si}$ wafer through the other small side. Neutron reflectivity curves were recorded with a fixed incident neutron beam in $\theta / 2 \theta$ geometry using a ${ }^{3} \mathrm{He}$ detector. Raw data were normalized to the number of incident neutrons and scaled as a function of momentum transfer, $Q=(4 \pi / \lambda) \sin \theta$ ( $\lambda$ is the neutron wavelength and $\theta$ is the angle of incidence). All measurement were performed at room temperature $\left(23^{\circ} \mathrm{C}\right)$. Prior to each run, the sample cell was rinsed with freshly prepared solution and the sample was equilibrated for at least $1 \mathrm{~h}$. The neutron reflectivity curves were analysed by fitting calculated reflectivity curves to the experimental data. Calculations were based on layer models for the interfacial structure and were carried out using the optical matrix method. ${ }^{39}$

\section{Results and discussion}

\section{Fluorescence correlation spectroscopy}

In Fig. 2, typical autocorrelation functions are shown that were derived from the fluorescence intensity fluctuations of BSA/ SPB suspensions. The autocorrelation functions were calculated as ${ }^{30,40}$

$$
G(\tau)=\frac{\langle\delta F(t) \cdot \delta F(t+\tau)\rangle}{\langle F(t)\rangle^{2}}
$$

where $\delta F(t)=F(t)-\langle F(t)\rangle$ is the fluorescence intensity fluctuation at time $t$, given as the deviation of the measured fluorescence intensity $F(t)$ from the time average $\langle F(t)\rangle$. If the fluorescence intensity fluctuations are caused by diffusion of
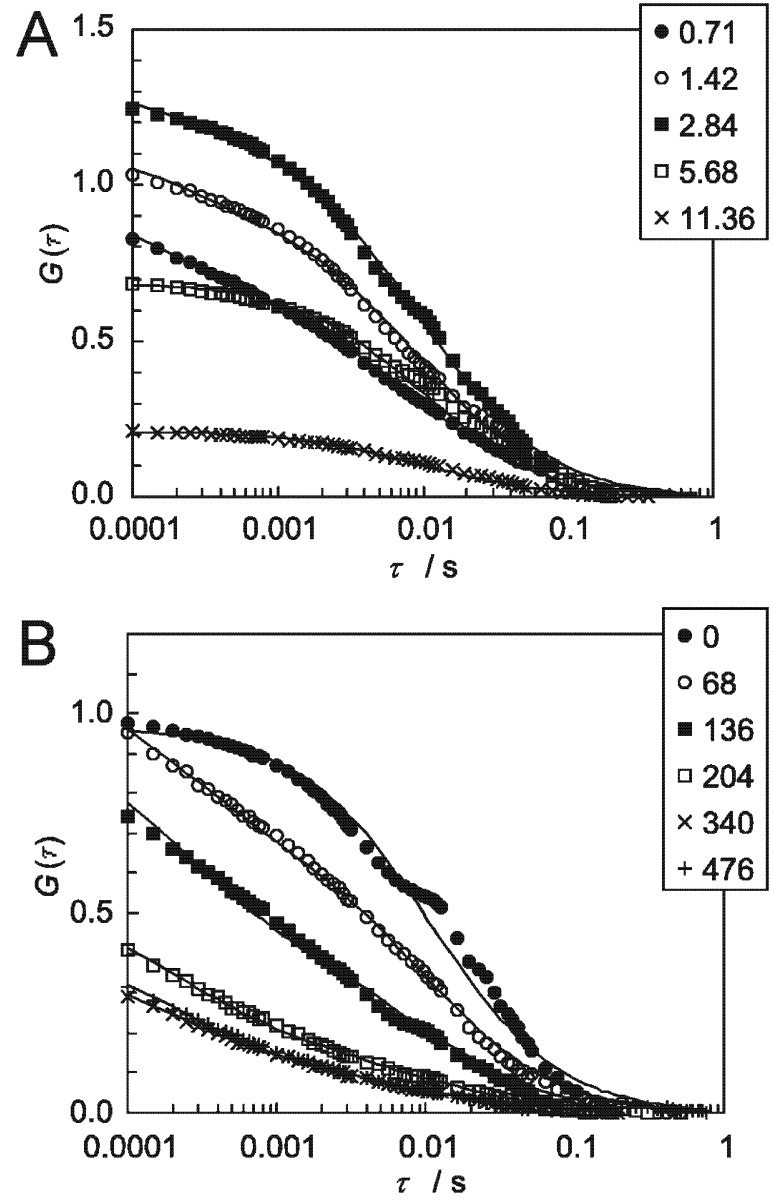

Fig. 2 Autocorrelation functions as calculated from observed fluorescence intensity fluctuations. (A) BSA/SPB suspensions containing 10 $\mu \mathrm{g}$ SPB and an increasing mass of BSA that is given in the inset in $\mu \mathrm{g}$. (B) BSA/SPB suspensions containing $20 \mu \mathrm{g} \mathrm{SPB}, 2.84 \mu \mathrm{g}$ BSA and an increasing concentration of $\mathrm{NaCl}$ that is given in the inset in $\mathrm{mM}$. The volume of the samples is $1.47 \mathrm{ml}$. The solid lines show global fits using two-component models.

two different fluorescent species into and out of the twophoton excitation volume, $G(\tau)$ can be described as

$$
\begin{aligned}
G(\tau)= & \left(\frac{\varepsilon_{1} N_{1}}{\varepsilon_{1} N_{1}+\varepsilon_{2} N_{2}}\right)^{2} \frac{\gamma}{N_{1}} g_{1}(\tau) \\
& +\left(\frac{\varepsilon_{2} N_{2}}{\varepsilon_{1} N_{1}+\varepsilon_{2} N_{2}}\right)^{2} \frac{\gamma}{N_{2}} g_{2}(\tau)
\end{aligned}
$$

where

$$
g_{i}(\tau)=\frac{1}{1+8 \mathrm{D}_{i} \tau / r_{0}^{2}} \cdot \frac{1}{\left(1+8 \mathrm{D}_{i} \tau / \omega_{0}^{2} r_{0}^{2}\right)^{1 / 2}}
$$

$\varepsilon_{i}$ is the molecular brightness, $N_{i}$ is the mean number of fluorescent particles within the excitation volume, and $\mathrm{D}_{i}$ is the diffusion constant of species $i . \omega_{0}=z_{0} / r_{0}$ is the ratio of the excitation volume dimensions parallel and perpendicular to the beam axis and $\gamma$ is a geometric factor depending on the shape of the excitation volume. As can be seen from eqn. (2), $G(\tau)$ of a two-component system is the sum of the individual autocorrelation functions weighted by the corresponding fractional intensities squared. A two-component analysis of $G(\tau)$ can be performed, when the diffusion constants $D_{1}$ and $D_{2}$ are different by a factor of about 1.6 provided the two components contribute comparably to the total fluorescence. ${ }^{30}$ Then, in addition to $\mathrm{D}_{1}$ and $\mathrm{D}_{2}$, the component amplitudes $G_{1}(0)$ and $G_{2}(0)$ can be extracted from the total autocorrelation function that contain the mean particle numbers of the two species in the two-photon excitation volume $\left[G_{1}(0)+G_{2}(0)=G(0)\right]$. 
The autocorrelation functions shown in Fig. 2 clearly indicate that BSA is binding to the SPB particles, since large changes in the amplitude of the curves are observed that cannot be explained assuming only a single diffusing species of non-adsorbed protein molecules. Thus, for a BSA/SPB suspension, two components can be identified that differ in their diffusion constant. The free non-adsorbed BSA molecules are the first component (index 1). They are characterized by a relatively large diffusion constant of $\mathrm{D}_{1}=60 \mu \mathrm{m}^{2} \mathrm{~s}^{-1}$. ${ }^{41}$ The second component (index 2) are the large and slowly diffusing spherical PAA brush particles with adsorbed fluorescent protein molecules. Therefore, the autocorrelation functions shown in Fig. 2 were analysed using a two-component model according to eqn. (2). Global fits were performed where the diffusion constant $D_{1}$ was fixed to $60 \mu \mathrm{m}^{2} \mathrm{~s}^{-1}$, whereas the diffusion constant $\mathrm{D}_{2}$ of the SPB particles was varied but linked across a data set. A value of $D_{2}=(1.6 \pm 0.2) \mu \mathrm{m}^{2} \mathrm{~s}^{-1}$ is recovered from the fits. The SPB particles have a core radius of $51 \mathrm{~nm}$ and a shell thickness of $61 \mathrm{~nm}$ in a $10 \mathrm{mM}$ salt solution. ${ }^{23}$ Using the Stokes-Einstein relationship these values lead to a diffusion constant of $1.9 \mu \mathrm{m}^{2} \mathrm{~s}^{-1}$, in favourable agreement with the experiment. No change in the diffusion constant of the SPB particles could be detected as the concentration of BSA was varied suggesting that the size of the SPB particles is not changing markedly when BSA is binding to them.

The component amplitude $G_{2}(0)$ of the SPB particles with adsorbed fluorescent BSA molecules is a measure for the degree of protein binding, since it increases when the brightness $\varepsilon_{2}$ of the SPB particles increases. $\varepsilon_{2}$ may approximately be expressed as

$$
\varepsilon_{2}=f \varepsilon_{1} N_{\mathrm{ads}} / N_{2}
$$

where $\varepsilon_{1}$ is the brightness of a non-adsorbed BSA molecule and $f$ is the change in brightness when the BSA molecule adsorbs at an SPB particle. $N_{\text {ads }}$ is the mean number of adsorbed protein molecules in the two-photon excitation volume and $N_{2}$ is the corresponding number of SPB particles. Then, $G_{2}(0)$ can be rewritten as

$$
\begin{aligned}
G_{2}(0) & =\left(\frac{\varepsilon_{2} N_{2}}{\varepsilon_{1} N_{1}+\varepsilon_{2} N_{2}}\right)^{2} \frac{\gamma}{N_{2}} \\
& =\frac{1}{\left[N_{1} /\left(N_{\text {ads }} f\right)+1\right]^{2}} \cdot \frac{\gamma}{N_{2}}
\end{aligned}
$$

where $N_{1}$ is the mean number of non-adsorbed BSA molecules in the two-photon excitation volume. From the analysis of the autocorrelation functions shown in Fig. 2, the component amplitude $G_{2}(0)$ was obtained. It is plotted in Figs. 3A and $3 \mathrm{~B}$ as a function of the $\mathrm{BSA}$ mass and the $\mathrm{NaCl}$ concentration, respectively.

As can be seen from Fig. 3A, with increasing total mass of BSA, $G_{2}(0)$ is passing through a maximum. The initial increase of $G_{2}(0)$ corresponds to an increase of the binding ratio $N_{\text {ads }} /$ $N_{1}$ (eqn. (5)) due to the adsorption of BSA at the SPB particles. On further addition of BSA, the SPB particles become saturated so that the number of non-adsorbed BSA molecules, $N_{1}$, increases stronger than the number of adsorbed BSA molecules, $N_{\text {ads }}$. Thus, $N_{\text {ads }} / N_{1}$ und $G_{2}(0)$ must decrease again at high BSA concentrations. In order to determine the number of adsorbed BSA molecules per SPB particle, the following equation can be used:

$$
\sqrt{G_{1}(0) N_{1} / \gamma}+\sqrt{G_{2}(0) N_{2} / \gamma}=1
$$

This equation is derived by adding the fractional intensities of the two components. For example, a sample containing $10 \mu \mathrm{g}$ SPB and $2.84 \mu \mathrm{g}$ BSA in $1.47 \mathrm{ml}$ yields an autocorrelation curve that is characterized by the component amplitudes $G_{1}(0)$ $=0.061$ and $G_{2}(0)=1.202$ (see Figs. 2A and 3A). The geometric factor $\gamma$ is 0.3535 and the size of the two-photon
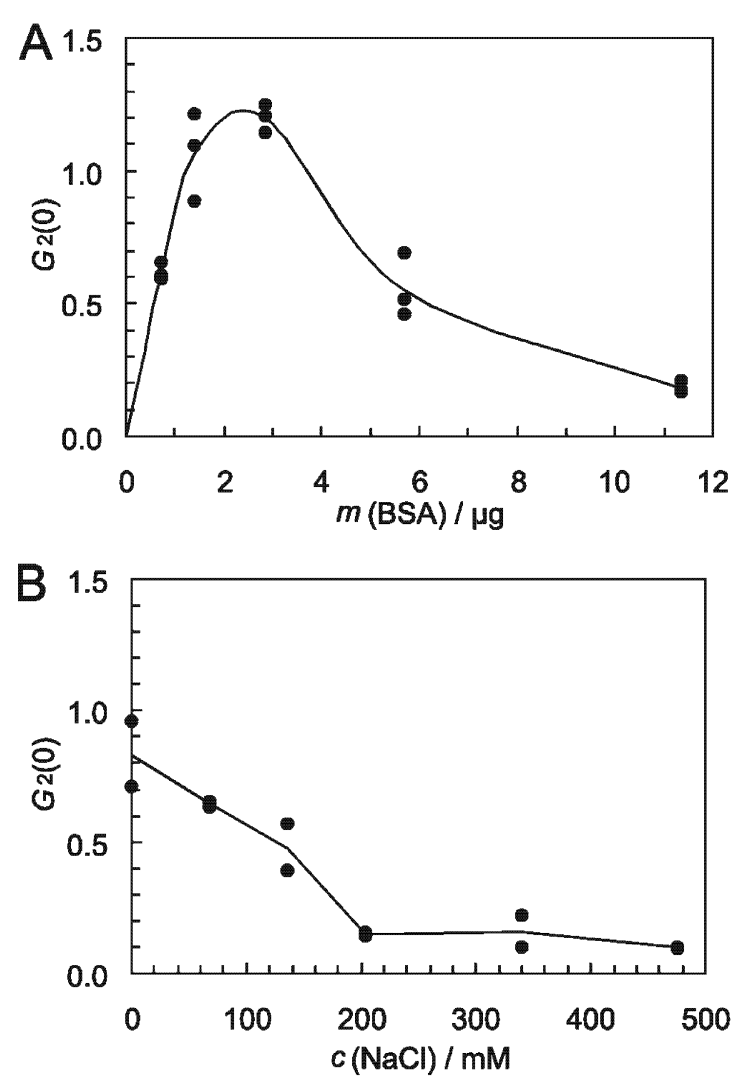

Fig. 3 Component amplitude of the SPB particles with adsorbed BSA molecules. Experimental conditions are the same as described in Fig. 2. Data series obtained in independent experiments are shown as symbols, the solid lines represent mean data values.

excitation volume can be calculated as $V=(\pi / 2)^{3 / 2} r_{0}{ }^{3} \omega_{0}=$ $1.22 \mathrm{fL}$ where $r_{0}$ and $\omega_{0}$ are known from calibration measurements (see Experimental section). ${ }^{42}$ With masses of $1.10 \times$ $10^{-19} \mathrm{~g}$ for a BSA molecule and $6.57 \times 10^{-16} \mathrm{~g}$ for an SPB particle, one obtains $N_{2}=0.013$ and thus $N_{1}=3.97$ (corrected for the degree a labelling). Since $2.84 \mu \mathrm{g}$ BSA per $1.47 \mathrm{ml}$ correspond to 21.4 BSA molecules per two-photon excitation volume, the number of adsorbed BSA molecules per SPB particle is $N_{\text {ads }} / N_{2}=1343$ which is equivalent to a BSA surface concentration of $1.8 \mathrm{mg} \mathrm{m}^{-2}$ (related to the area of the PAA brush mid-plane of the SPB particles). This value is relatively high. For example, only about $0.75 \mathrm{mg} \mathrm{m}^{-2}$ are reported for BSA adsorbed on silica particles at $\mathrm{pH}=7$ and $10 \mathrm{mM} \mathrm{NaCl}{ }^{43}$ Thus, a huge amount of BSA is binding to a PAA brush under electrostatic repulsion condition (both the BSA and the PAA are negatively charged at $\mathrm{pH}=6.1$ ).

More interestingly, when the concentration of $\mathrm{NaCl}$ in a $\mathrm{BSA} / \mathrm{SPB}$ suspension is raised to a few hundred $\mathrm{mM}$, the component amplitude $G_{2}(0)$ is decreasing towards 0 (Fig. 3B) which can only be explained by a decreasing brightness $\varepsilon_{2}$ of the SPB and a decreasing binding ratio $N_{\text {ads }} / N_{1}$ (eqn. (5)). This salt-induced protein resistance of the spherical PAA brush particles is somewhat surprising, since at elevated ionic strength the electrostatic repulsion between the BSA molecules and the PAA brush is lowered. Indeed, when proteins bind to other interfaces under electrostatic repulsion condition, higher surface concentrations are observed at higher ionic strength. ${ }^{43} 45$

The reason for the observed salt-induced protein resistance of a PAA brush will be discussed below.

\section{Neutron reflectometry}

Neutron reflectometry was applied to analyse the degree of BSA binding to a planar PAA brush and to determine the volume fraction profile of BSA normal to the interface at a low 
and high ionic strength. In Fig. 4, neutron reflectivity curves of an $\mathrm{Si} / \mathrm{d}-\mathrm{PS} / \mathrm{PS}-\mathrm{PAA} /$ solution interface, i.e. a planar PAA brush, are shown with $\mathrm{D}_{2} \mathrm{O}$ used as the solvent. Very similar curves were obtained when using $\mathrm{HDO}$ instead of $\mathrm{D}_{2} \mathrm{O}$. Pronounced oscillations are observed for the $\mathrm{D}_{2} \mathrm{O}$ buffer sample, since the PS-PAA molecules are not deuterated and have a lower scattering length density than $\mathrm{D}_{2} \mathrm{O}$. When the buffer solution is replaced by a BSA solution $\left(0.05 \mathrm{mg} \mathrm{ml}^{-1}\right)$ in the presence of $500 \mathrm{mM} \mathrm{NaCl}$, a neutron reflectivity curve is obtained that is essentially overlapping with that of the buffer solution suggesting that the scattering length density profile of the interface did not change. Thus, BSA is not binding to the planar PAA brush at an elevated $\mathrm{NaCl}$ concentration of 500 $\mathrm{mM}$, although repulsive electrostatic interactions between the negatively charged BSA and the negatively charged PAA are screened under this condition. However, rinsing the sample cell with a BSA solution of the same concentration but without added $\mathrm{NaCl}$ leads to significant changes in the neutron reflectivity curve (Fig. 4A). This observation shows that, when lowering the ionic strength of the solution, BSA is binding to the PAA brush under electrostatic repulsion conditions.

To analyse the structure of the interface, a three-layer model was assumed which represents the most simple but physically reasonable model. The first layer accounts for the d-PS film on the Si wafer (index 1), the second layer covers the binding region of the PS-PAA copolymer to the d-PS film (index 2), and the third layer represents the PAA brush with or without adsorbed BSA (index 3). The Si wafer and the sample solution
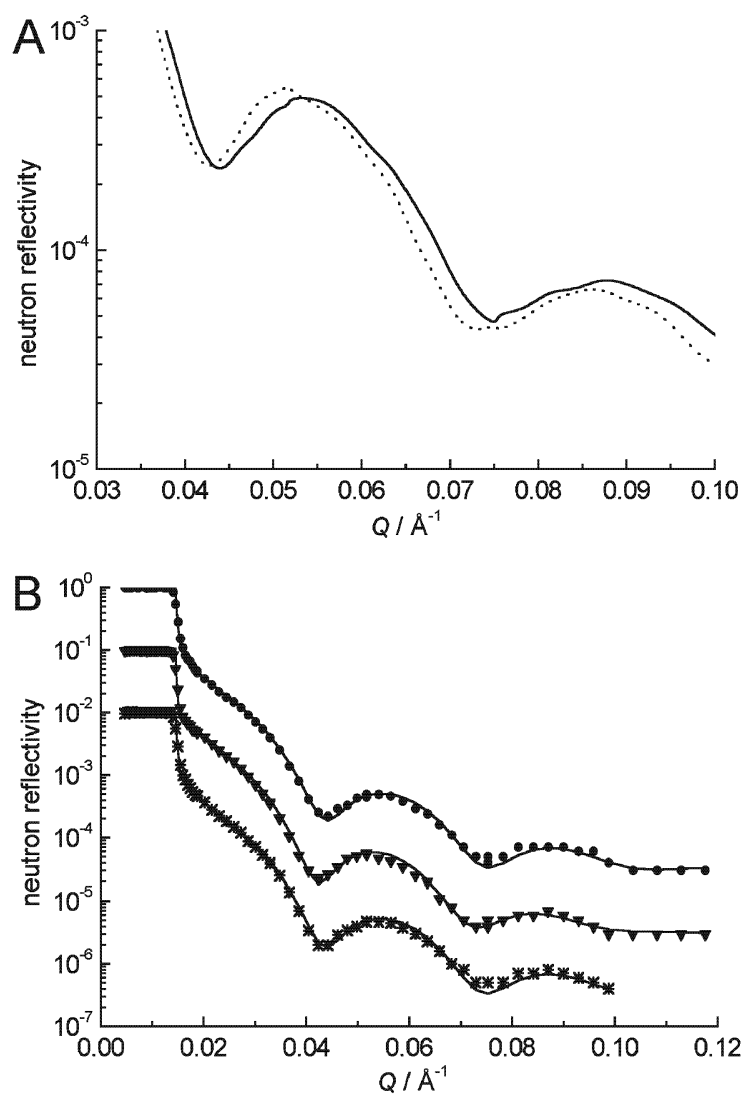

Fig. 4 Neutron reflectivity curves of an Si/d-PS/PS-PAA/solution interface, i.e. a planar PAA brush. In diagram A, curves are shown over a limited $Q$-range only to illustrate the difference between the data obtained with a buffer solution (solid line) and a BSA/buffer solution without added $\mathrm{NaCl}$ (dotted line). In diagram $\mathrm{B}$, the measured reflectivities are shown over the whole $Q$-range as symbols, whereas the solid lines represent fits on the basis of a layer model. The data of diagram B refer to a buffer solution (circles), a BSA/buffer solution (triangles, shifted by a factor of 0.1 ), and a BSA/buffer solution with $500 \mathrm{mM} \mathrm{NaCl}$ (asterisks, shifted by a factor of 0.01 ). Without shift, the latter data are essentially overlapping with those of the buffer solution. will be denoted with index 0 and index 4 , respectively. The three-layer model is completely characterized by three types of parameters: the scattering length density, the layer thickness and the interlayer roughness. By varying these parameters, the model was fitted globally to pairs of reflectivity curves differing in contrast due to the different solvent used ( $\mathrm{D}_{2} \mathrm{O}$ or $\left.\mathrm{HDO}\right)$. In the global fitting, the layer thicknesses and the interlayer roughnesses were linked across a data pair, i.e., these values were varied simultaneously which largely reduces the number of scattering length density profiles consistent with the measured neutron reflectivity curves. The scattering length densities of the Si wafer, the d-PS film and the sample solutions were not varied in the fitting procedure and set to $\rho_{0}=2.07 \times 10^{-6}$ $\AA^{-2}, \rho_{1}=6.42 \times 10^{-6} \AA^{-2}$, and to $\rho_{4}=6.37 \times 10^{-6} \AA^{-2}\left(\mathrm{D}_{2} \mathrm{O}\right)$ or to $\rho_{4}=4.64 \times 10^{-6} \AA^{-2}$ (HDO). ${ }^{46}$ To account for an $\mathrm{NaCl}$ solution concentration of $500 \mathrm{mM}$, the latter two values were increased by $0.04 \times 10^{-6} \AA^{-2}$. In Table 1 , the fitting results are summarized and the corresponding calculated neutron reflectivity curves are shown in Fig. $4 \mathrm{~B}$ as solid lines.

As can be seen from Table 1, the binding of BSA to the PAA brush can be modelled by reducing the scattering length density $\rho_{3}$ and increasing the layer thickness $d_{2}$. This change in the scattering length density profile can be related to the volume fraction profile of BSA across the interface. At any distance $z$ from the Si interface we may approximately express the total scattering length density by the scattering length densities of the BSA, the polymer (index pol) and the solution (index sol):

$$
\rho^{\prime \prime}(z)=\rho_{\mathrm{BSA}} \phi_{\mathrm{BSA}}+\rho_{\mathrm{pol}} \phi_{\mathrm{pol}}+\rho_{\mathrm{sol}}\left(1-\phi_{\mathrm{BSA}}-\phi_{\mathrm{pol}}\right)
$$

where $\phi_{i}$ is the volume fraction of component $i$ at distance $z$. In the absence of BSA, this scattering length density reduces to

$$
\rho^{\prime}(z)=\rho_{\text {pol }} \phi_{\text {pol }}+\rho_{\text {sol }}\left(1-\phi_{\text {pol }}\right)
$$

Here it is assumed that the binding of BSA to the PAA brush only involves a replacement of water by protein molecules. Then, we may write:

$$
\phi_{\mathrm{BSA}}(z)=\frac{\rho^{\prime \prime}(z)-\rho^{\prime}(z)}{\rho_{\mathrm{BSA}}-\rho_{\mathrm{sol}}}
$$

where $\rho_{\text {BSA }}=3.19 \times 10^{-6} \AA^{-2}$ (in $\mathrm{D}_{2} \mathrm{O}$ ) and $\rho_{\text {sol }}=\rho_{4}$. Thus, we can calculate the volume fraction profile of BSA by subtracting the scattering length density profile of the buffer sample from that of the BSA solution.

In Fig. 5, the volume fraction profile of BSA is shown in the absence of $\mathrm{NaCl}$ and at an $\mathrm{NaCl}$ concentration of $500 \mathrm{mM}$. Without added $\mathrm{NaCl}$, the profile clearly shows that BSA is penetrating deeply into the PAA brush. A maximum BSA concentration is located at the inner brush interface. This observation suggests a higher PS-PAA chain density in this region which may be a result of the method applied for the preparation of the planar PAA brush (see Experimental section). Indeed, a more homogeneous protein distribution within a PAA brush has been found for spherical polyelectrolyte brushes. ${ }^{47}$ The penetration of BSA into the PAA brush is related to the binding mechanism of BSA involving counterions (see below). It also leads to a relatively high amount of adsorbed BSA, since the packing of BSA molecules at the interface is extended to the third dimension along the PAA chains. The adsorbed mass of BSA per surface area is given by the integration of the volume fraction profile according to:

$$
\Gamma=\frac{M}{V} \int_{0}^{\infty} \phi_{\mathrm{BSA}}(z) \mathrm{d} z
$$

where $M=66267 \mathrm{~g} \mathrm{~mol}^{-1}$ is the molar mass and $V=48574$ $\mathrm{cm}^{3} \mathrm{~mol}^{-1}$ is the molar volume of BSA. ${ }^{33}$ One can calculate a BSA surface concentration of $\Gamma=1.5 \mathrm{mg} \mathrm{ml}^{-1}$ at a planar PAA brush in the absence of $\mathrm{NaCl}$ which is roughly the same as that found on the spherical PAA brush particles (see above). 
Table 1 Scattering length density profiles of an $\mathrm{Si} / \mathrm{d}-\mathrm{PS} / \mathrm{PS}-\mathrm{PAA} /$ solution interface $^{a}$

\begin{tabular}{|c|c|c|c|c|c|c|c|c|}
\hline Solution & $\rho_{0} / 10^{-6} \AA^{-2}$ & $\rho_{1} / 10^{-6} \AA^{-2}$ & $\rho_{2} / 10^{-6} \AA^{-2}$ & $\rho_{3} / 10^{-6} \AA^{-2}$ & $\rho_{4} / 10^{-6} \AA^{-2}$ & $d_{1} / \AA$ & $d_{2} / \AA$ & $d_{3} / \AA$ \\
\hline Buffer & $(2.07)$ & $(6.42)$ & 5.06 & 6.18 & $(6.37)$ & 169 & 45 & 165 \\
\hline $0.05 \mathrm{mg} \mathrm{ml}^{-1} \mathrm{BSA}$ & $(2.07)$ & $(6.42)$ & 5.06 & 6.04 & $(6.37)$ & 169 & 58 & 152 \\
\hline $0.05 \mathrm{mg} \mathrm{ml}^{-1} \mathrm{BSA}+500 \mathrm{mM} \mathrm{NaCl}$ & $(2.07)$ & $(6.42)$ & 5.06 & 6.18 & $(6.41)$ & 169 & 47 & 163 \\
\hline
\end{tabular}

${ }^{a}$ Parameters in parentheses were not varied in the fitting procedure. Interlayer roughnesses are $\sigma_{01}=13 \AA, \sigma_{12}=10 \AA, \sigma_{23}=8 \AA, \sigma_{34}=80 \AA$. The scattering length densities are given for samples with $\mathrm{D}_{2} \mathrm{O}$ as the solvent.

However, at $500 \mathrm{mM} \mathrm{NaCl}$, the BSA volume fraction within the planar PAA brush is almost zero (Fig. 5) indicating that the planar PAA brush becomes protein-resistant at an elevated ionic strength of a few $100 \mathrm{mM}$, although the electrostatic repulsion between the negatively charged $\mathrm{BSA}$ and PAA is lowered under this condition. This finding is in agreement with the results of the FCS experiments at the spherical PAA brush particles described above.

The unusual salt dependence of the BSA binding capacity of a PAA brush represents a unique effect that has been explained by a new type of driving force for protein adsorption. ${ }^{23}$ Since protein resistance of a PAA brush is observed at high ionic strength where electrostatic interactions are screened, van der Waals and hydrophobic interactions do not appear to be of major significance. However, the latter type of interactions may explain in part a residual BSA adsorption at high ionic strength (see Figs. 3B and 5). Reducing the ionic strength of the protein solution leads to an enhanced electrostatic repulsion between BSA and PAA, yet the degree of BSA binding to a PAA brush is increasing. It has been concluded that a release of counterions is the dominant driving force for the binding of proteins to a PAA brush, since a huge number of $\mathrm{H}^{+}$and $\mathrm{Na}^{+}$ counterions are trapped in the PAA brush at low ionic strength. ${ }^{23}$ A protein molecule that carries multiple positive charges can act as multivalent counterion and a corresponding number of monovalent counterions is released. ${ }^{48}$ This effect increases the entropy of the system and reduces the osmotic pressure within the PAA brush. On the other hand, a negatively charged protein molecule has several positively charged counterions in a low ionic strength solution. Within the PAA brush, most of these counterions will be released due to the enormous excess of PAA counterions. ${ }^{31}$ Thus, counterions are released in either case. However, at elevated ionic strength of the protein solution, this driving force vanishes rendering a PAA brush protein resistant. ${ }^{31}$ In addition, approaching protein molecules are repelled from the interface by steric interactions with the PAA chains.

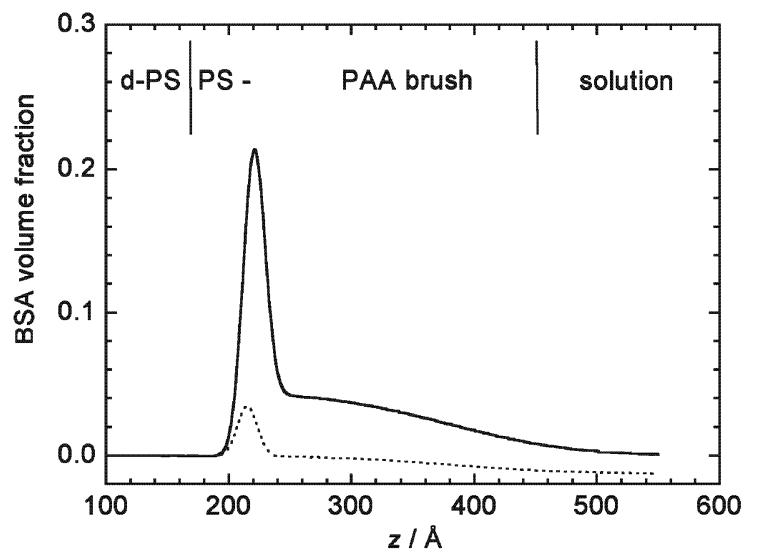

Fig. 5 Volume fraction of BSA across an Si/d-PS/PS-PAA/solution interface. The solid line corresponds to a solution of $0.05 \mathrm{mg} \mathrm{ml}^{-1}$ BSA, the dotted line was obtained when $500 \mathrm{mM} \mathrm{NaCl}$ was added to the BSA solution.

\section{Conclusions}

The degree of BSA binding to PAA brushes has been studied in situ by using two different techniques: two-photon excitation FCS and neutron reflectometry. The experiments performed in this study show that both spherical and planar PAA brushes bind large and similar amounts of BSA at low ionic strength under electrostatic repulsion conditions. However, when the ionic strength of the protein solution is raised to a few $100 \mathrm{mM}$ and the electrostatic repulsion is lowered, the PAA brushes were found to become protein resistant. These findings are in favourable agreement with an earlier study where the irreversible adsorption of BSA at spherical PAA brush particles was measured ex situ. ${ }^{23}$ All investigations suggest that a PAA brush represents a unique surface coating with a salt-dependent protein affinity that cannot be explained by simple electrostatic screening effects. Rather, a release of counterions will be the main driving force for protein adsorption at a PAA brush. Since this counterion release is no more effective at a high ionic strength of the protein solution, the protein affinity of a PAA brush is vanishing at these conditions. It is worthwhile to note that a "switching" of the protein affinity of a PAA brush by changing the ionic strength of a protein solution in the range of a few $100 \mathrm{mM}$ mainly leaves the structure and dynamics of the protein molecules unaffected.

\section{Acknowledgements}

We thank the Deutsche Forschungsgemeinschaft (DFG) and the Hahn-Meitner-Institut Berlin for financial support. The fluorescence experiments reported in this paper were performed at the Laboratory for Fluorescence Dynamics (LFD) at the University of Illinois at Urbana-Champaign (UIUC). The LFD is supported jointly by the National Center for Research Resources of the National Institutes of Health (PHS 5 P41-RRO3155) and UIUC.

\section{References}

1 Biopolymers at interfaces, ed. M. Malmsten, Marcel Dekker, New York, 2003.

2 Physical chemistry of biological interfaces, ed. A. Baszkin and W. Norde, Marcel Dekker, New York, 2000.

3 B. Kasemo, Curr. Opin. Solid State Mater. Sci., 1998, 3, 451-459.

4 S. R. Weinberger, T. S. Morris and M. Pawlak, Pharmacogenomics, 2000, 1, 395-416.

5 W. Norde, Macromol. Symp., 1996, 103, 5-18.

6 C. M. Roth and A. M. Lenhoff, Langmuir, 1995, 11, 3500-3509.

7 C. M. Roth, B. L. Neal and A. M. Lenhoff, Biophys. J., 1996, 70, 977-987.

8 G. Jackler, R. Steitz and C. Czeslik, Langmuir, 2002, 18 6565-6570.

9 C. Czeslik, Z. Phys. Chem., 2004, 218, 771-801.

10 J. Monahan and J. J. Wilker, Langmuir, 2004, 20, 3724-3729.

11 S. I. Jeon, J. H. Lee, J. D. Andrade and P. G. de Gennes, J. Colloid Interface Sci., 1991, 142, 149-158.

12 S. I. Jeon and J. D. Andrade, J. Colloid Interface Sci., 1991, 142, 159-166.

13 I. Szleifer, Biophys. J., 1997, 72, 595-612.

14 E. P. K. Currie, W. Norde and M. A. Cohen Stuart, Adv. Colloid Interface Sci., 2003, 100-102, 205-265.

15 J. G. Archambault and J. L. Brash, Colloids Surf., B, 2004, 33 111-120. 
16 E. Österberg, K. Bergström, K. Holmberg, T. P. Schuman, J. A. Riggs, N. L. Burns, J. M. van Alstine and J. M. Harris, J. Biomed. Mater. Res., 1995, 29, 741-747.

17 J. Piehler, A. Brecht, K. Hehl and G. Gauglitz, Colloids Surf., B, $1999,13,325-336$.

18 E. Ostuni, R. G. Chapman, R. E. Holmlin, S. Takayama and G. M. Whitesides, Langmuir, 200 1, 17, 5605-5620.

19 R. S. Kane, P. Deschatelets and G. M. Whitesides, Langmuir, $2003,19,2388-2391$.

20 Z. Zhang, B. Menges, R. B. Timmons, W. Knoll and R. Förch, Langmuir, 2003, 19, 4765-4770.

21 R. L. C. Wang, H. J. Kreuzer and M. Grunze, J. Phys. Chem. B, 1997, 101, 9767-9773.

22 S. Herrwerth, W. Eck, S. Reinhardt and M. Grunze, J. Am. Chem. Soc., 2003, 125, 9359-9366.

23 A. Wittemann, B. Haupt and M. Ballauff, Phys. Chem. Chem. Phys., 2003, 5, 1671-1677.

$24 \mathrm{X}$. Guo and M. Ballauff, Langmuir, 2000, 16, 8719-8726.

25 X. Guo and M. Ballauff, Phys. Rev. E, 2001, 64, 51406.

26 A. Wittemann and M. Ballauff, Anal. Chem., 2004, 76, 2813-2819.

27 T. Neumann, B. Haupt and M. Ballauff, Macromol. Biosci., 2004, 4, 13-16.

28 G. Jackler, A. Wittemann, M. Ballauff and C. Czeslik, Spectroscopy, 2004, 18, 289-299.

29 C. Czeslik, R. Jansen, M. Ballauff, A. Wittemann, C. A. Royer, E. Gratton and T. Hazlett, Phys. Rev. E, 2004, 69, 21401.

30 S. T. Hess, S. Huang, A. A. Heikal and W. W. Webb, Biochemistry, 2002, 41, 697-705.

31 C. Czeslik, G. Jackler, R. Steitz and H.-H. von Grünberg, J. Phys. Chem. B, 2004, 108, 13395-13402.
32 T. P. Russell, Mater. Sci. Rep., 1990, 5, 171-271.

33 D. C. Carter and J. X. Ho, Adv. Protein Chem., 1994, 45 153-203.

34 X. Guo, A. Weiss and M. Ballauff, Macromolecules, 1999, 32, 6043-6046.

35 W. Kern, J. Electrochem. Soc., 1990, 137, 1887-1892.

36 E. P. K. Currie, A. B. Sieval, M. Avena, H. Zuilhof, E. J. R. Sudhölter and M. A. Cohen Stuart, Langmuir, 1999, 15 7116-7118.

37 Langmuir-Blodgett Films, ed. G. Roberts, Plenum Press, New York, 1990.

38 F. Mezei, R. Golub, F. Klose and H. Toews, Physica B, 1995, 213/ 214, 898-900.

39 W. N. Hansen, J. Opt. Soc. Am., 1968, 58, 380-390.

40 K. M. Berland, P. T. C. So and E. Gratton, Biophys. J., 1995, 68, 694-701.

41 M. Placidi and S. Cannistraro, Europhys. Lett., 1998, 43 476-481.

42 P. Schwille, U. Haupts, S. Maiti and W. W. Webb, Biophys. J., $1999,77,2251-2265$.

43 S. Robinson and P. A. Williams, Langmuir, 2002, 18, 8743-8748.

44 G. Ladam, C. Gergely, B. Senger, G. Decher, J.-C. Voegel, P. Schaaf and F. J. G. Cuisinier, Biomacromolecules, 2000, 1 $674-687$.

45 M. Malmsten, D. Muller and B. Lassen, J. Colloid Interface Sci., 1997, 193, 88-95.

46 http://www.ncnr.nist.gov/resources/n-lengths/list.html.

47 S. Rosenfeldt, A. Wittemann, M. Ballauff, E. Breininger, J. Bolze and N. Dingenouts, submitted.

48 C. Fleck and H. H. von Grünberg, Phys. Rev. E, 2001, 63, 61804. 